\title{
Mid-IR images of methanol masers and ultracompact HII regions
}

\author{
Paolo Persi ${ }^{1}$, Mauricio Tapia ${ }^{2}$ and Anna Rosa Marenzi ${ }^{1}$ \\ ${ }^{1}$ INAF/IASF-Roma Via fosso del Cavaliere,100, oo133 Roma-Italy \\ email: paolo.persi@iasf-roma.inaf.it \\ ${ }^{2}$ IA-UNAM Ensenada, Mexico
}

Methanol masers and UCHII regions trace massive star formation sites. We have undertaken a mid-IR survey of 17 regions containing methanol masers and UCHIIs in order to locate the young stellar sources associated with them. The images were obtained from 8.7 to $18.8 \mu \mathrm{m}$ with the mid-IR camera CID (Salas et al. 2003) on the $2.1 \mathrm{~m}$ telescope of the Observatorio Astronomico Nacional at San Pedro Martir (Baja California, Mexico). The images were taken with a scale $0.55 "$ / pix and the mean PSF was 1.5-2.0" (FWHM) close to the diffraction limit. We report as an example in Fig. 1 (left panel) our $18.8 \mu \mathrm{m}$
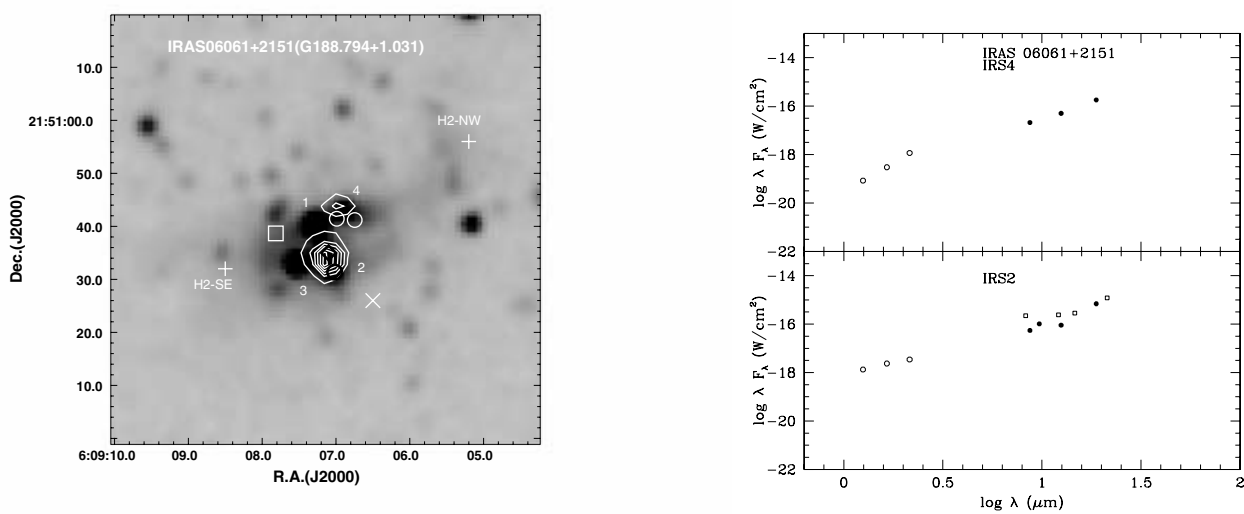

Figure 1. Left: $K s$ image of IRAS $06061+2151$ with the $18.8 \mu \mathrm{m}$ contours. Right: SED of the source \#2 and \#4 associated with the UCHIIs.

contours of IRAS $06061+2151$ superimposed to the 2MASS $K s$ image. A young cluster of at least 4 sources has been found centered on the IRAS source (Anandarao et al. 2004). We have found two mid-IR sources coinciding with the source \#2 and \#4 of Anandarao et al.(2004). The source \#4 is at the center of two $\mathrm{H} 2$ knots and a high velocity molecular outflow. The mid-IR emission from \#2 is extended and coincides with the UCHII and MSX source. The methanol maser is approximately 10" south of the source \#2. The SEDs of both sources are illustrated in Fig. 1 (right panel). The IR spectral indices of source $\# 2$ and $\# 4$ are $\alpha(\mathrm{IR})=1.9$ and 2.2 respectively.

\section{References}

Anandarao, B.G., Chakraborty, A., Ojha, D.K., \& Testi, L. 2004, A\& A 421, 1045

Salas, L., Gutierrez, L., Tapia, M., et al. 2003, in: Instrument Design and Performance for Optical/Infrared Telescopes, SPIE Proc. 4941, 594 